\title{
Die Feinstruktur des Integumentes und der Muskelansatzstellen von Echiniscoides sigismundi (Heterotardigrada)
}

\author{
H. Greven \& G. Grohé \\ Abteilung Histophysiologie des Zoologischen Institutes der Universität Münster; \\ Münster (Westf.), \\ und \\ Institut für Meereskunde an der Christian-Albrechts-Universität Kiel; \\ Kiel, Bundesrepublik Deutschland
}

\begin{abstract}
The fine structure of the integument and the muscle attachments in Echiniscoides sigismundi (Heterotardigrada). The structure of the integument and the muscle attachments of the marine heterotardigrade E. sigismundi (M. SCHULTZE) was studied by electron microscopy. The cuticle consists of several layers: an outer tripartite (or multilayered) epicuticle, perhaps with an outermost coat; a homogeneous inner epicuticle; a trilaminated layer; an intracuticle; and a fibrous procuticle. These features resemble the cuticle described in Eutardigrada; in contrast, areas on the legs and near the claws, with an outer multilayered epicuticle and a striated layer (inner epicuticle), are - as far as investigated - more similar to the cuticle in Heterotardigrada. The epidermis consists of a single cell layer without glands. The muscle attachments are in line with the general pattern described in the eutardigrade Macrobiotus bufelandi and in Arthropoda.
\end{abstract}

\section{EINLEITUNG}

Elektronenmikroskopische und histochemische Untersuchungen am TardigradenIntegument haben gezeigt, daß es aus einer einschichtigen Epidermis und einer komplexen Cuticula besteht, die zudem beträchtliche morphologische Unterschiede zwischen den beiden Ordnungen Hetero- und Eutardigraden aufweist.

Innerhalb der Heterotardigraden sind bisher die Integumente von Vertretern der Halechiniscidae (Greven, 1975), Batillipedidae (Kristensen, mdl. Mitclg.) und Echiniscidae (Crowe et al., 1970; Greven, 1971a, b, 1972; Bussers \& Jeuniaux, 1973a; SchUSTER et al., 1975), innerhalb der Eutardigraden von Vertretern der Macrobiotidae (Baccetti \& Rosati, 1971; Crowe et al., 1971a, b; Greven, 1971b, 1972, 1975; Bussers \& Jeuniaux, 1973a, b; Shaw, 1974) und Milnesiidae (Greven, 1972; Bussers \& Jeuniaux, 1973a, b; Dewel \& Clark, 1973a, b) licht- und elektronenmikroskopisch bearbeitet worden. 
Uber die chemische Zusammensetzung der Cuticula liegen bis auf wenige mit Hilfe lichtmikroskopischer Methoden gewonnene Daten detailliertere Ergebnisse nur für die Eutardigraden-Cuticula vor. Einzelne Cuticula-Lagen, wie eine epidermisnahe chitinhaltige Procuticula und eine außen liegende mehrfach geschichtete Epicuticula ohne Chitin erinnern an die Verhältnisse bei Arthropoden (vgl. hierzu die Diskussionen bei Baccetti \& Rosatr, 1971; Greven, 1972; Bussers \& Jeuniaux, 1973a, b). Ein Vergleich der Cuticulae von Vertretern der Hetero- und Eutardigraden ist daher z. Z. nur auf Grund morphologischer und topographischer Kriterien möglich (GREvEN, 1972, 1975). Diese Vergleiche führten zu einer weitgehend neutralen Bezeichnung der verschiedenen Zonen innerhalb der Tardigraden-Cuticula, die sich teilweise an der für die Arthropoden-Cuticula üblichen Terminologie orientiert (GReven, 1975). Im günstigsten Fall, z. B. bei Milnesium tardigradum (Eutardigrada) lassen sich folgende Cuticula-Schichten identifizieren: Außere Epicuticula mit Schleimschicht, innere Epicuticula, Dreifachlage, Intracuticula mit distinkter innerer Begrenzung, granuläre Lage und Procuticula.

Auf Grund aller bisher vorliegenden Befunde (Literaturübersicht bei Greven, 1975 ) ist es noch nicht möglich, Verallgemeinerndes über Vorkommen, Fehlen oder strukturelle Abweichungen bestimmter Cuticula-Schichten bei verschiedenen Tardigradenordnungen oder-familien auszusagen.

Im folgenden beschreiben wir erstmals die Feinstruktur des Integuments und der Muskelansatzstellen des marinen Tardigraden Echiniscoides sigismundi (M. ScHultzE) aus der Familie Oreellidae, die innerhalb der Echiniscoidea nur mit ungepanzerten Arten vertreten ist (Marcus, 1929; RamazzotTI, 1972).

\section{MATERIAL UND METHODEN}

Die Versuchstiere wurden auf Helgoland oberhalb des Felswatts in Höhe der Hochwasserlinie gesammelt (zur Sammeltechnik vgl. GroHé, in Vorb.) bei $4^{\circ} \mathrm{C}$ in $3,5 \%$ igem Glutaraldehyd in $0,01 \mathrm{~m}$ Phosphatpuffer, $\mathrm{pH} 7,6$, vorfixiert $(1,5 \mathrm{~h})$, anschließend im gleichen Puffer mit einem Zusatz von $7,5 \%$ Saccharose gespült, in $2 \%$ iger Osmiumsäure nachfixiert $(1,5 \mathrm{~h})$ und in Araldit eingebettet.

Die Dünnschnitte wurden mit Glasmessern auf den Reichert Ultramikrotomen Om U 2 und U 3 hergestellt, $z$. T. ohne Verwendung von Folien auf Kupfernetze aufgezogen, mit Uranylacetat und/oder Bleicitrat nach Reynolds kontrastiert (RurHMANN, 1966) und in den Elmiskopen EM 101 (Siemens) (Leihgabe der Stiftung Volkswagenwerk) und 9-S2 (Zeiss) untersucht. Die Abbildung 1 verdanken wir Herrn Dr. G. Uhurg (Biologische Anstalt Helgoland).

\section{BEFUNDE}

Echiniscoides sigismundi lebt zwischen Algen, besonders Enteromorpha, im obersten Litoral (vgl. GroHÉ, in Vorb.). Seine Größe schwankt zwischen 200 und $300 \mu \mathrm{m}$. Die Cuticula ist glatt und hyalin. Cirrus lateralis, Clava, fünf bis neun 
untereinander gleichartige Krallen und Fehlen der Vasa malpighii kennzeichnen ihn als Heterotardigraden (Abb. 1, Marcus, 1929; RamazzotTr, 1972).

\section{Die Cuticula}

Dorsale und ventrale Cuticula von Echiniscoides sigismundi zeigen in ihrer Feinstruktur keine Unterschiede.

Die Epicuticula. Die Kontaktzone zur Umwelt bildet die äußere Epicuticula. Sie besteht in den meisten Fällen aus zwei elektronendichten Bändern, die von einer transparenten Zone getrennt werden (Abb. 2a, c, d). Die Breite dieser drei Lagen beträgt etwa 120 Å. Bisweilen kann die äußere Epicuticula auch mehrschichtig sein; ihre Dicke beträgt dann etwa 200 bis $250 \AA . A$ Ob ihr noch osmiophile Substanzen in Form einer Schleimschicht aufgelagert sind (vgl. BACCETTI \& ROSATI, 1971) können wir aus unseren Aufnahmen nicht eindeutig entnehmen. Allerdings muß festgehalten werden, daß die Untersuchungen oft durch das Anhaften von Algen, Detritus und kleinen Steinchen an der Cuticula erschwert werden. Die innere Epicuticula schließt mit einer Dicke von etwa 350 bis $1500 \AA$ an. Sie ist aus lockerem, fädigem Material aufgebaut und hebt sich bei der Präparation leicht ab (Abb. 2).

Die Dreif a chlage. Unter der Epicuticula ist eine wellig verlaufende Dreifachlage zu erkennen; auch hier werden zwei dunkle Zonen von einer helleren getrennt; ihre Dicke beträgt etwa $150 \AA$ (Abb. 2b).

Die Intracuticula. Der Lage nach entspricht die nächste Schicht der Intracuticula (BACCetTi \& Rosati, 1971; Greven, 1975). Sie ist etwa 0,1 bis 0,2 $\mu \mathrm{m}$ dick, ziemlich osmiophil und deutlich von der darunterliegenden Procuticula abgesetzt (Abb. 2a, b, c). Bei Tieren, die sich in der Häutungsphase befinden und bereits Epicuticula, Dreifachlage und Procuticula gebildet haben, ist die Intracuticula nur unvollkommen als mäßig dunkle Zone unter der Dreifachlage zu erkennen (Abb. 2d).

Die Procuticula. Den größten Raum innerhalb der Cuticula nimmt die Procuticula mit einer Dicke von 0,3 bis $0,8 \mu \mathrm{m}$ ein. Sie besteht aus fibrillärem Material

Abb. 1: Echiniscoides sigismundi, dorsal. Beachte die homonyche Bekrallung und die Divertkel

$$
\text { des Mitreldarms }(300: 1)
$$

Abb. 2: Die Rumpfcuticula von Ebiniscoides sigismundi. a Epicuticula (E), Intracuticula (I), Procuticula $(P)$. Die äußere Epicuticula $\rightarrow$ ) ist dreischichtig $(14700: 1)$. b Dreifachlage (D) unterhalb der Epicuticula (E) (120000:1). c Stark gewellte Cuticula mit osmiophilem Material in der Procuticula $(\rightarrow)(14700: 1)$. d Neu angelegte Cuticula (Häutungstier). Die Intracuticula ist kaum erkennbar $(\rightarrow)(40000: 1)$

\begin{tabular}{llll} 
& \multicolumn{3}{c}{ Abkürzungen in den Bildlegenden } \\
äE & äußere Epicuticula & $\mathrm{K}$ & Kralle \\
$\mathrm{B}$ & Basallamelle & $\mathrm{L}$ & Lipidtropfen \\
$\mathrm{Cu}$ & Cuticula & $\mathrm{Mi}$ & Mitochondrium \\
$\mathrm{D}$ & Dreifachlage & $\mathrm{Mu}$ & Muskelzelle \\
$\mathrm{E}$ & Epicuticula & $\mathrm{N}$ & Zellkern \\
$\mathrm{Ep}$ & Epidermis & $\mathrm{P}$ & Procuticula \\
$\mathrm{H}$ & Hämocoel & RER & rauhes endoplasmatisches \\
$\mathrm{I}$ & Intracuticula & & Reticulum
\end{tabular}



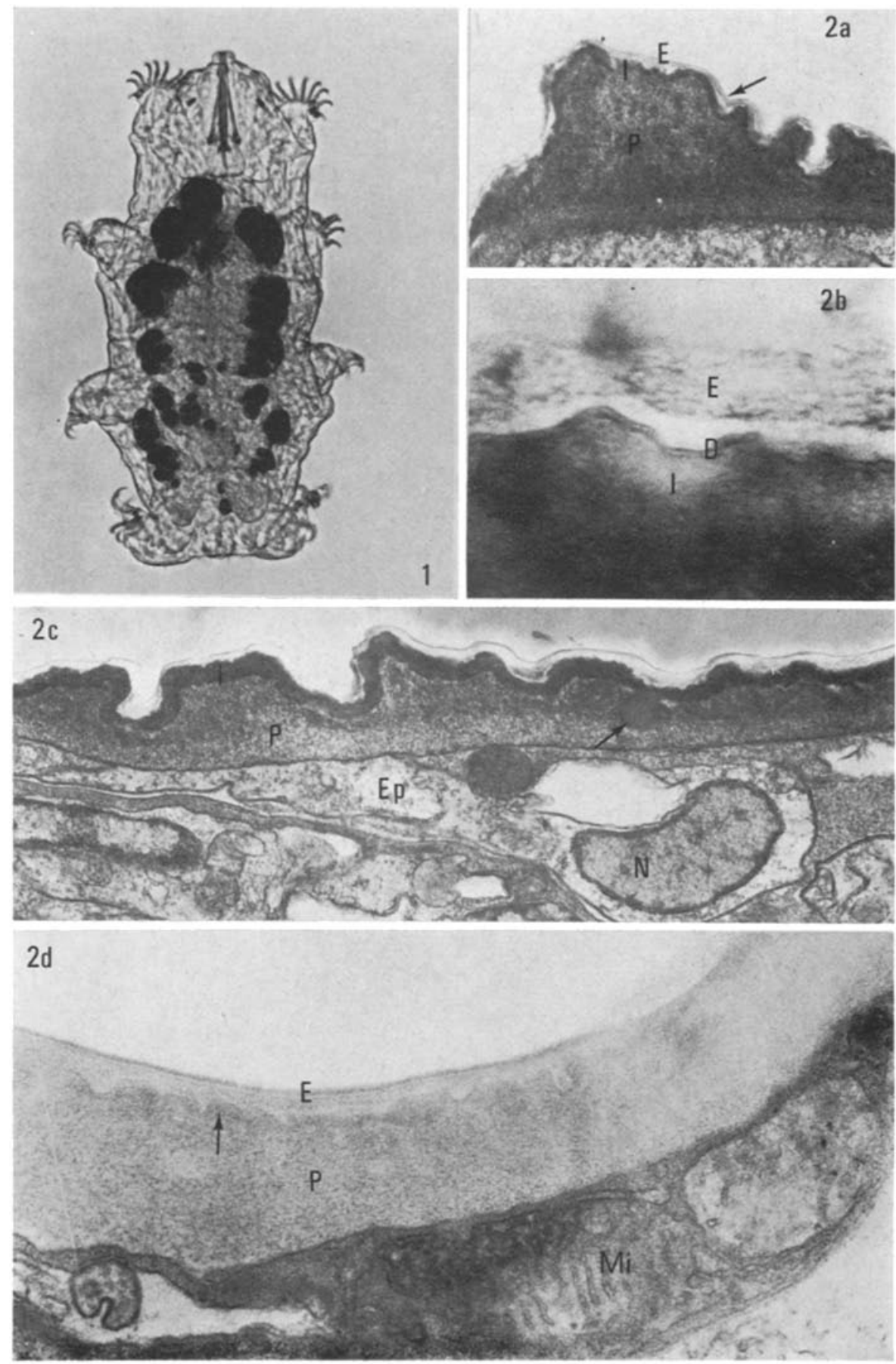
und ist relativ elektronendicht (Abb. $2 a, c, 5 a$ ). In ihr liegt bisweilen Material, daß der Substanz der Intracuticula ähnelt (Abb. 2c).

In der Cuticula der Beinregion sind innerhalb der Epicuticula beträchtliche morphologische Abwandlungen festzustellen. Die äußere Epicuticula ist deutlich mehrschichtig. In der inneren Epicuticula treten parallel zueinander angeordnete Stäbchen mit einer Periode von ca. $150 \AA ̊$ auf, die im Querschnitt ein poly-oder hexagonales Muster zeigen. Sie setzen an der innersten dunklen Lage der äußeren Epicuticula an und reichen fast bis an die Dreifachlage, ohne aber mit dieser eine Verbindung einzugehen (Abb. 3).

\section{Die Epidermis}

Die Epidermiszellen schließen mit dem apikalen Plasmalemm an die Procuticula an (Abb. 4). Die Epidermis ist einschichtig, von variabler Dicke - an manchen Stellen mißt sie nur $0,15 \mu \mathrm{m}$ - und sendet keine Fortsätze in die Cuticula hinein. Die lateralen Zellgrenzen sind mäßig gewunden.

Im Cytoplasma befinden sich rauhes endoplasmatisches Reticulum, freie Ribosomen, Mitochondrien, manchmal von Zisternen des rauhen ER umhüllt, und der Kern, dessen Hülle mit Ribosomen besetzt ist. Vereinzelt treten Cytosomen, Lipidtropfen und osmiophiles Material auf, das in seinem Aufbau dem Cuticula-Material ähnelt. Den Abschluß zum Hämocoel bildet eine dünne Basallamelle (Abb. 4). Drüsenzellen konnten wir nicht finden.

Damit ähnelt die Epidermis von E. sigismundi in ihrem Aufbau ganz der anderer daraufhin untersuchter Eu- und Heterotardigraden.

\section{Die Muskelansatzstellen}

Die somatischen aus jeweils einer langgestreckten Zelle bestehenden Muskeln der Tardigraden (MARCUS, 1929; SHAW, 1974; WALZ, 1974) inserieren oft zu mehreren an der Cuticula.

Muskelansatzstellen haben bisher Crowe et al. (1971) für den Eutardigraden Macrobiotus areolatus abgebildet mit dem Hinweis, daß ihre Struktur den entsprechenden Stellen bei Arthropoden ähnelt. Genauere Untersuchungen an dem verwandten Macrobiotus bufelandi bestätigten diese Ergebnisse (SHAw, 1974).

Bei E. sigismundi ist an der Muskelansatzstelle die Cuticula nicht wesentlich verdickt. Allerdings ragen cuticulare Fortsätze in die darunterliegende Epidermiszelle (Abb. 5a, b, c). In Bündeln angeordnete Filamente durchziehen die Cuticula vorwie-

Abb. 3: Die Cuticula der Beine von Echiniscoides sigismundi. a "Gestreifte "Epicuticula am Krallenansatz (40000:1). 6 Mehrschichtige äußere Epicuticula (äE) und Streifenmuster oberhalb des Krallenansatzes (75 000:1)

Abb. 4: Epidermiszelle mit Mitochondrium (Mi), rauhem endoplasmatischem Reticulum (RER), Lipidtropfen (L) und Zellkern (N) $(24000: 1)$ 

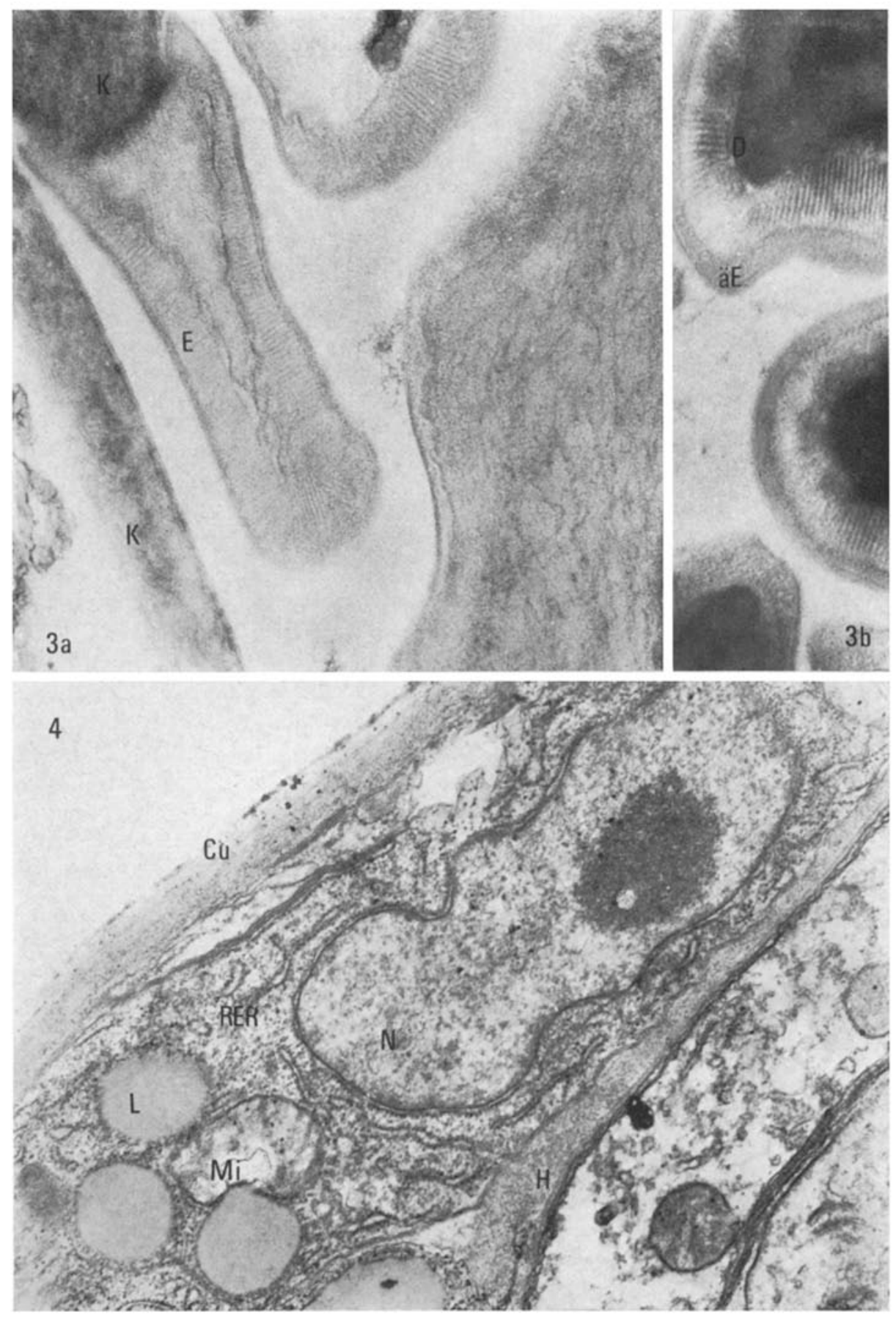
gend in vertikaler Richtung; an der Peripherie der Ansatzstelle verlaufen sie jedoch in einem Bogen durch die Cuticula. Die Filamente durchziehen innere Epicuticula, Intra- und Procuticula und einen Teil der cuticularen Fortsätze, die weit in die Epidermiszelle hineinragen (Abb. 5a, b, c). Von hier aus zieht sich ähnlich fibrilläres Material durch die außerordentlich dünne und onganellenarme Epidermiszelle und endet in dem basal liegenden mit elektronendichtem Material angefüllten Bereich ("Verstärkungs$z^{a n} e^{c c}$ ) (Abb. 5a, b, c). Innerhalb der Epidermiszelle haben wir keine Mikrotubuii identifizieren können.

Das basale Plasmalemm der Epidermiszelle verläuft stark gewellt, ebenso das nahe unter ihr liegende Sarkolemm der Muskelzelle, an das sich ebenfalls eine elektronendichtere Zone anschließt (Abb. 5b, c, d). Zwischen Epidermis- und Muskelzelle liegt eine relativ homogene, mäßig elektronendichte Schicht, die den Einfaltungen der Zellmembranen nur wenig folgt und sich am Rande der Muskelansatzstellen in die Basallaminae der beiden Zellen aufspaltet (Abb. 5b, c). Das gilt auch für die entsprechende Zone zwischen zwei oder mehreren Muskelzellen (Abb. 5d).

\section{DISKUSSION}

Bei den bisher untersuchten Heterotandigraden ist die Cuticula nicht einheitlich aufgebaut. Die gepanzerten Echiniscus-Arten besitzen dorsal eine stark verdickte innere Epicuticula mit einem komplizierten Hohlraumsystem. Ventral gliedert sich diese in feine vertikal verlaufende Kanälchen („striated layer“) und darunterliegende Säulen auf. Weiterhin fallen feine, die dorsale Intracuticula durchziehende Filamente auf (Crowe et al., 1970; Greven, 1971a, 1972, 1975). Die dorsale und ventrale Cuticula der marinen Arten Halechiniscus (Greven, 1975) und Batillipes (Kristensen, mdl. Mittlg.) ähneln bis auf geringfügige Unterschiede in der "striated layer" der ventralen Cuticula der Echiniscidae. Eine innere Begrenzung der Intracuticula und die granuläre Schicht fehlen wahrscheinlich. Die bisher allen Heterotardigraden gemeinsame drei- bis mehrschichtige äußere Epicuticula, die wellig verlaufende Dreifachlage und die fibrilläre Procuticula sind morphologisch mit den entsprechenden Schichten der Eutardigraden-Cuticula zu vergleichen (GREven, 1972, 1975).

Innerhalb der Eutandigraden zeigt die Cuticula einen einheitlicheren Aufbau (vgl. die Zusammenfassung bei Greven, 1975). Die von Baccettr \& Rosatr (1971) für Macrobiotus bufelandi beschriebenen zahlreichen Cuticula-Schichten (s. Einleitung) sind von Nachuntersuchern allerdings eindeutig nur bei Milnesium tardigradum identifiziert worden (GREvEN, 1975; vgl, auch SHAw, 1974). Bei Macrobiotus areolatus und den bisher untersuchten Isobypsibius-Arten fehlen wahrscheinlich die innere

Abb. 5: Die Muskelansatzstellen von Echiniscoides sigismundi, a Gekrümmt und vertikal verlaufende cuticulare Filamente $(\rightarrow)$, die z. T. die Epicuticula durchdringen $(\leftrightarrow)$ und sich auch in die Epidermiszelle fortzusetzen scheinen $(\rightarrow)(28500: 1)$. b Cuticulare Fortsätze erreichen die elektronendichtere Zone an der Basis der Epidermiszelle. Plasmalemm und Sarkolemm sind stark gewellt. Dazwischen liegt eine dicke Basallamelle (B) (30000:1). c Schrägschnitt mit quergetroffenen cuticularen Zapfen (30000:1). d Ansatzstellen von zwei Muskelzellen. Die dicke Basallamelle spaltet sich auf $(\rightarrow)(30000: 1)$ 


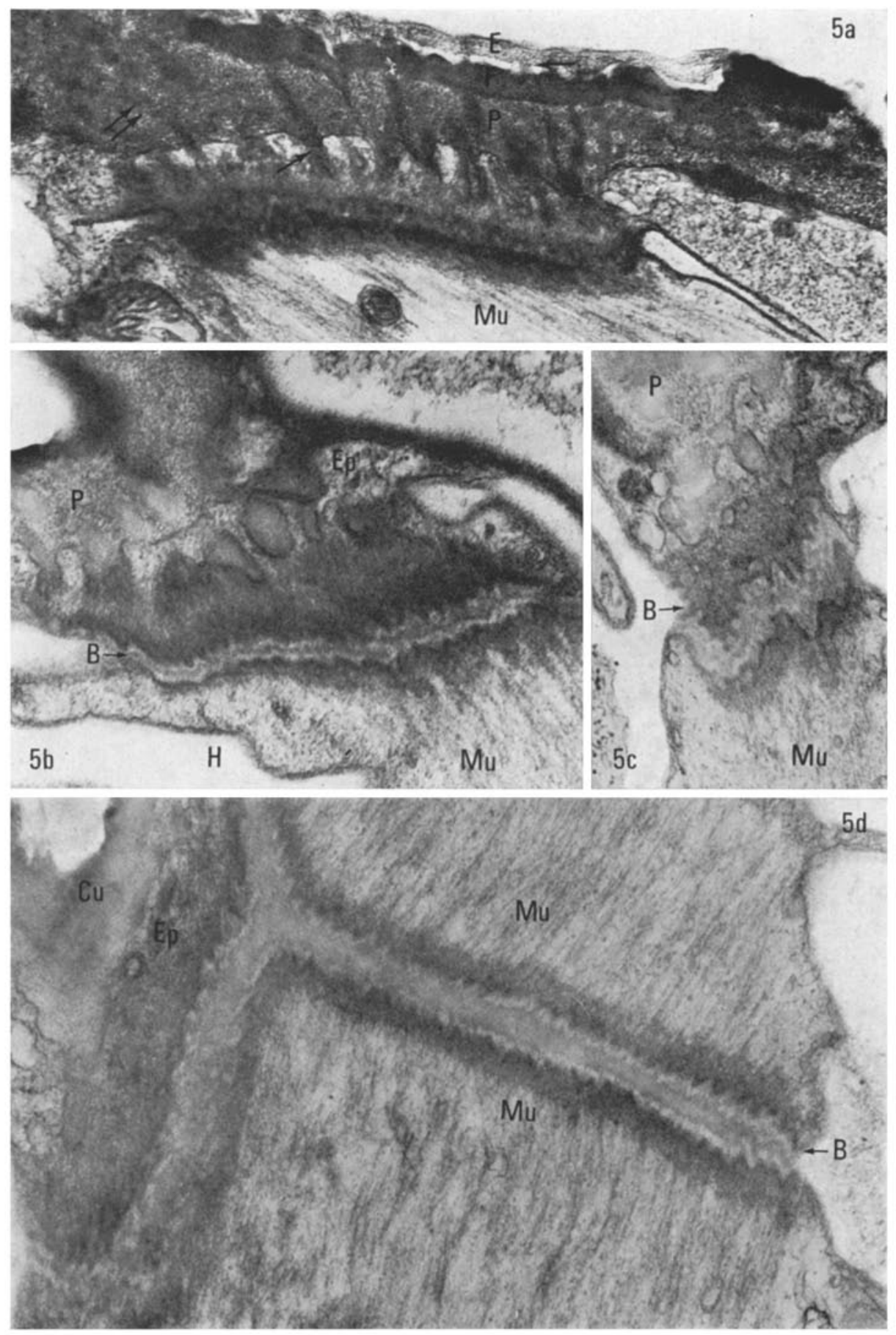


Abgrenzung der Intracuticula und die sich anschließende granuläre Lage. Möglicherweise ist der bei diesen Arten als Intracuticula bezeichnete elektronendichtere $\mathrm{Be}$ reich unter der Dreifachlage nur ein modifizierter Teil der Procuticula.

Ventrale und dorsale Cuticula von $E$. sigismundi stimmen nicht nur im Aufbau überein, die Cuticula zeigt darüber hinaus Merkmale, die bisher nur von der Eutardigraden-Cuticula bekannt waren: eine nicht auffallend strukturierte innere Epicuticula und eine homogene Intracuticula. Andererseits erinneren Cuticula-Areale im Bereich der Extremitäten an die Verhältnisse, wie sie bisher nur für Heterotardigraden beschrieben wonden sind: Auflösung der inneren Epicuticula in ein Streifenmuster („striated layer"). Unterhalb dieser Streifen schließt sich unmittelbar die Dreifachlage an. Eine weitere Aufgliederung der inneren Epicuticula in Säulen unterhalb des Streifensaums wie z. B. bei Echiniscus-Arten (Crowe et al., 1970; Greven, 1971a, 1972) und Batillipes (Kristensen, mdl. Mittlg.) unterbleibt. Das erinnert an Cuticula-Bezirke bei Halechiniscus spec. (GREven, 1975).

Der morphologische Aufbau der Muskelansatzstellen stimmt im wesentlichen mit dem der entsprechenden Stellen bei Macrobiotus bufelandi überein (SHAw, 1974). Die cuticularen Filamente (zur Terminologie vgl. SHAw, 1974) entspringen bei $M$. bufelandi an der Intracuticula und durchziehen die Procuticula z. T. bis in die zapfenartigen Vorstülpungen in die Epidermis, die hemidesmosomenartige Strukturen besitzen (SHAw, 1974). Bei E. sigismundi ziehen diese cuticularen Filamente bis an die äußere Epicuticula, die an diesen Stellen häufig geringfügig eingesenkt ist. CAvENEY (1969) konnte bei apterygoten Insekten zeigen, daß die "muscle attachment fibres“ die Pro- und Epicuticula passieren, aber nicht direkt an der äußeren Epicuticula (cuticulin layer) ansetzen.

Zwischen Epidermiszelle und Muskelzelle verläuft bei E. sigismundi ein homogenes Band, das sich an den Enden der Muskelansatzstelle in die beiden Basallaminae aufspaltet. SHAw (1974) hält diese Zone, die bei M. bufelandi sehr viel inhomogener ist, in Anlehnung an die Befunde von BaccetTr \& Rosatr (1969), die kollagenhaltige Laminae um die inneren Organe von $M$. bufelandi beschrieben haben, für eine modifizierte Basallamelle bzw. Bindegewebe. Eine derartig ausgeprägte Basallamina kommt offensichtlich bei Arthropoden nicht vor. Vielleicht ist sie mit der „intercellular matrix" zwischen Muskel- und Epidermiszelle bei verschiedenen Apterygoten vergleichbar (CAveney, 1969). Generell läßt sich sagen, daß die Struktur der Muskelansatzstellen bei den bisher untersuchten Tandigraden (Heterotardigrada: E. sigismundi; diese Arbeit; Eutardigrada: Macrobiotus bufelandi; SHAw, 1974) weitgehend dem Arthropodenschema entsprechen. Die Struktur dieser Bezirke ist innerhalb der Arthropoden bis auf geringfügige Unterschiede (z. B. Fehlen von Mikrotubuli in den Epidermiszellen von Milben, verschiedenartige Ausbildung von Desmosomen und Hemidesmosomen) überraschend einheitlich (vgl. u. a. LAIFOoK, 1967; CAvENEY, 1969; SMITH et al., 1969; Kuo et al., 1971; weitere Literatur und funktionelle Deutung bei SHAw, 1974).

Daher könnten diese und andere feinstrukturelle aber auch histochemische Befunde (Cuticula, Kollagen, Spermien) als Hinweise für die stammesgeschichtliche Affinität der Tardigraden zu den Arthropoden gedeutet werden (vgl. auch die Diskussionen bei Baccettr \& Rosatr, 1969, 1971; BaccetTI et al., 1971; Bussers \& 
JeUniaux, 1973a, b; GREVEN, 1972; SHAw 1974), obgleich auch in neuerer Literatur eine Verwandtschaft mit den Aschelminthen diskutiert wird (CRowe et al., 1970, 1971; Dewel \& Clark, 1973a, b). Gewichtigere Argumente für die eine oder andere Auffassung werden sich wahrscheinlich erst nach der neuerlichen Untersuchung der Tardigraden-Embryologie anführen lassen.

\section{ZUSAMMENEASSUNG}

1. Das Integument des marinen Heterotardigraden Echiniscoides sigismundi besteht aus einer Cuticula und einer einschichtigen Epidermis ohne Drüsen.

2. Die Rumpfcuticula besitzt eine drei- bis mehrschichtige äußere Epicuticula (vielleicht mit einer aufgelagerten Schleimschicht), eine nicht auffallend strukturierten inneren Epicuticula, eine Dreifachlage, eine homogenen Intracuticula und eine Procuticula. Sie zeigt damit Merkmale, die bisher nur von der EutardigradenCuticula bekannt waren.

3. Epicuticula-Bezirke der Beine und des Krallenansatzes lassen eine mehrschichtige äußere und eine in "Streifen“ aufgelöste innere Epicuticula erkennen. Diese Strukturen sind bisher allen Heterotardigraden-Cuticulae gemeinsam.

4. Die Feinstruktur der Muskelansatzstellen ist mit entsprechenden Stellen bei dem Eutardigraden Macrobiotus bufelandi zu vergleichen und paßt in das allgemeine für die Arthropoden geltende Schema.

\section{ZITIERTE LITERATUR}

BacCEtTI, B. \& Rosati, F., 1969. Electron microscopy on tardigrades. 1. Connective tissue. J. submicrosc. Cytol. 1, 197-205.

- -1971 . Electron microscopy on tardigrades. III. The integument. J. Ultrastruct. Res. 34, 214-243.

- \& SELMI, G., 1971. Electron microscopy on tardigrades. 4. The spermatozoon. Monitore zool. ital. 5, 231-240.

Bussers, J. C. \& Jeuniaux, C., 1973a. Chitinous cuticle and systematic position of Tardigrada. Biochem. Syst. 1, 77-78.

- 1973b. Structure et composition de la cuticule de Macrobiotus sp. et de Milnesium tardigradum (Tardigrades). Annls Soc. zool. Belg. 103, 271-279.

Cavenex, S., 1969. Muscle attachment related to cuticle architecture in Apterygota. J. Cell Sci. 4, 541-559.

Crowe, J. H., Newerk, I. M. \& Tromson, W. W., 1970. Echiniscus viridis (Tardigrada): Fine structure of the cuticle. Trans. Am. microsc. Soc. 89, 316-325.

$-\ldots-1971$. Fine structure and chemical composition of the cuticle of the tardigrade, Macrobiotus areolatus MURRay. J. Microsc. 11, 107-120.

- - - 1971b. Cuticle formation in the tardigrade, Macrobiotus areolatus Murray. J. Microsc. 11, 121-132.

Dewel, R. A. \& Clark, W. H., 1973a. Studies on the tardigrades. I. Fine structure of the anterior foregut of Milnesium tardigradum DoYere. Tissue Cell 5, 133-146.

- - 1973b. Studies on the tardigrades. II. Fine structure of the pharynx of Milnesium tardigradum DOYERE. Tissue Cell 5, 147-159. 
Greven, H., 1971a. Zur Feinstruktur der inneren Epicuticula von Echiniscus testudo. Naturwissenschaften 58, 367-368.

- 1971b. Zur Morphologie der Tardigraden. Rasterelektronenmikroskopische Untersuchungen an Macrobiotus hufelandi und Echiniscus testudo. Forma functio 4, 283-302.

- 1972. Vergleichende Untersuchungen am Integument von Hetero- und Eutardigraden. Z. Zellforsch. mikrosk. Anar. 135, 517-558.

- 1975. New results and considerations regarding the fine structure of the cuticle in tardigrades. Memorie Ist. ital. Idrobiol. 32 (Suppl.), 113-131.

GroHÉ, G. Die Verbreitung von Echiniscoides sigismundi (Tardigrada) in der EnteromorphaZone von Helgoland. (In Vorbereitung.)

Kuo, J. S., McCully, M. E. \& HagGis, G. H., 1971. The fine structure of muscle attachments in an acarid mite Caloglyphus mycophagus (MEGNin) (Acarine). Tissue Cell 3, 605-613.

LA1-Fook, J., 1967. The structure of developing muscle insertions in insects. J. Morph. 123, 503-528.

Marcus, E., 1929. Tardigrada. Bronn's Kl. Ordn. Tierreichs 5 (Abr. 4, 3).

Ramazzotti, G., 1972. Il phylum Tardigrada. Memorie Ist, ital. Idrobiol. 28, 1-732.

Ruthmann, A., 1966. Methoden der Zellforschung. Franckh, Stuttgart, 301 pp.

Schuster, R. O., Grigarick, A. A. \& ToftNer, E. C., 1975. Ultrastructure of the tardigrade cuticle. Memorie Ist. ital. Idrobiol. 32 (Suppl.), (in press).

SHAw, K., 1974. The fine structure of muscle cells and their attachments in the tardigrade Macrobiotus hufelandi. Tissue Cell 6, 431-445.

SMTth, D. S., Järlfors, U. R. \& Russel, F. E., 1969. The fine structure of muscle attachments in a spider (Latrodectus mactans FABR.) Tissue Cell 1, 673-687.

Warz, B., 1974. The fine structure of somatic muscles of Tardigrada. Cell Tiss. Res. 149, $81-89$.

Anschrift des erstgenannten Autors: Dr. H. Greven

Abteilung für Histophysiologie

Zoologisches Institut der Universität

D - 44 Münster

Hüfferstr. 1

Bundesrepublik Deutschland 